\title{
Design Concepts for an Outage Control Center Information Dashboard
}

\author{
Jacques Hugo \\ Shawn St. Germain \\ Ronald Farris \\ Cheradan Thompson \\ McKenzie Whitesides
}

December 2015

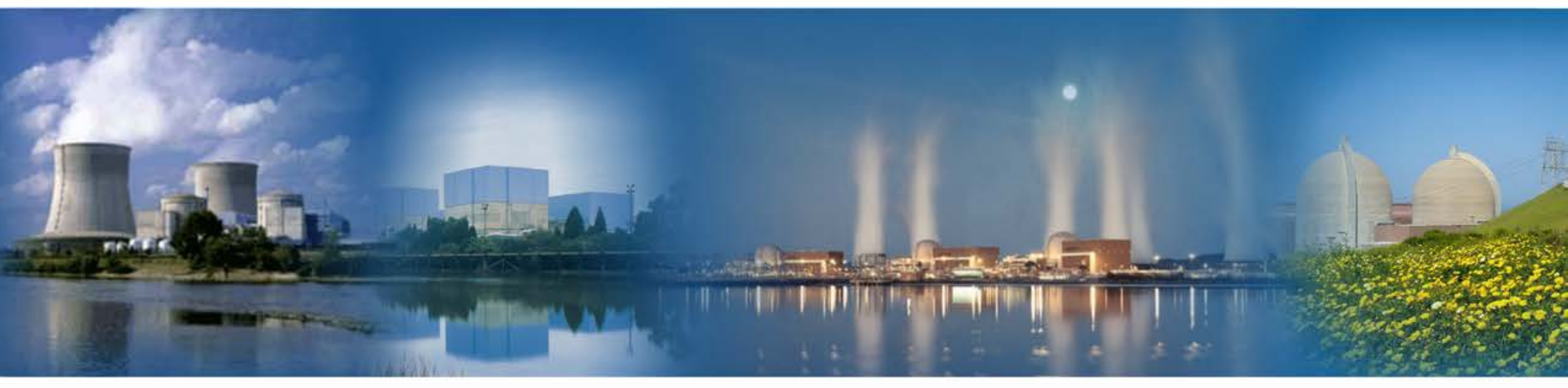

The INL is a U.S. Department of Energy National Laboratory operated by Battelle Energy Alliance 


\section{DISCLAIMER}

This information was prepared as an account of work sponsored by an agency of the U.S. Government. Neither the U.S. Government nor any agency thereof, nor any of their employees, makes any warranty, expressed or implied, or assumes any legal liability or responsibility for the accuracy, completeness, or usefulness, of any information, apparatus, product, or process disclosed, or represents that its use would not infringe privately owned rights. References herein to any specific commercial product, process, or service by trade name, trade mark, manufacturer, or otherwise, does not necessarily constitute or imply its endorsement, recommendation, or favoring by the U.S. Government or any agency thereof. The views and opinions of authors expressed herein do not necessarily state or reflect those of the U.S. Government or any agency thereof. 


\title{
Design Concepts for an Outage Control Center Information Dashboard
}

\author{
Jacques Hugo \\ Shawn St. Germain \\ Ronald Farris \\ Cheradan Thompson \\ McKenzie Whitesides
}

December 2015

\author{
Idaho National Laboratory \\ Idaho Falls, Idaho 83415 \\ http://www.inl.gov
}

Prepared under Work Package L-12N060305

Prepared for the

U.S. Department of Energy

Office of Nuclear Energy

Under DOE Idaho Operations Office

Contract DE-AC07-05ID14517 



\section{EXECUTIVE SUMMARY}

The nuclear industry, and the business world in general, is facing a rapidly increasing amount of data to be dealt with on a daily basis. In the last two decades, the steady improvement of data storage devices and means to create and collect data along the way influenced the manner in which we deal with information. Most data is still stored without filtering and refinement for later use. Many functions at a nuclear power plant generate vast amounts of data, with scheduled and unscheduled outages being a prime example of a source of some of the most complex data sets at the plant. To make matters worse, modern information and communications technology is making it possible to collect and store data faster than our ability to use it for making decisions. However, in most applications, especially outages, raw data has no value in itself; instead, managers, engineers and other specialists want to extract the information contained in it. The complexity and sheer volume of data could lead to information overload, resulting in getting lost in data that may be irrelevant to the task at hand, processed in an inappropriate way, or presented in an ineffective way. To prevent information overload, many data sources are ignored so production opportunities are lost because utilities lack the ability to deal with the enormous data volumes properly. Decision-makers are often confronted with large amounts of disparate, conflicting and dynamic information, which are available from multiple heterogeneous sources. Information and communication technologies alone will not solve this problem. Utilities need effective methods to exploit and use the hidden opportunities and knowledge residing in unexplored data resources. Superior performance before, during and after outages depends upon the right information being available at the right time to the right people. Acquisition of raw data is the easy part; instead, it is the ability to use advanced analytical, data processing and data visualization methods to turn the data into reliable information and comprehensible, actionable information. Techniques like data mining, filtering and analysis only work reliably for well-defined and well-understood problems. The path from data to decision is more complex. The ability to communicate knowledge during outages and emergent issues is crucial. This paper presents an approach to turn the unused data into an opportunity: applying principles from semiotics, human factors and visual analytics to transform the traditional way of processing outage data into media that will improve the collective situation awareness, knowledge, decisions, actions and overall performance of the entire outage team, and also support the reliability, quality and overall effectiveness of maintenance work. The application of the proposed visualization methods will become the medium of a semi-automated analytical process where humans and machines cooperate using their respective, distinct capabilities for the most effective results.

Key Words: Advanced Nuclear Power Plant, Operational Concept, Concept of Operations, Human Factors 
THIS PAGE INTENTIONALLY BLANK 


\section{CONTENTS}

EXECUTIVE SUMMARY

iii

ACRONYMS

Error! Bookmark not defined.

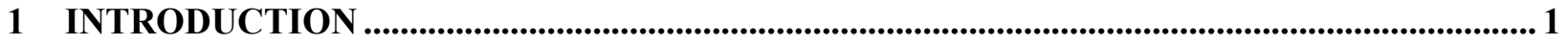

1.1 Outage Information and Situation Awareness ................................................................. 1

1.2 A semiotic approach to the design of an advanced OCC Information Display........................2

2 Data complexity and the use of information dashboards................................................................... 3

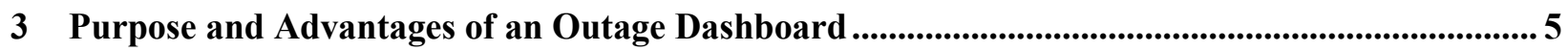

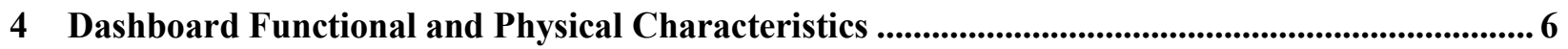

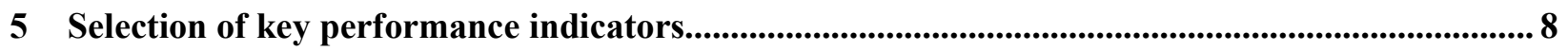

6 Basic Dashboard Design Concepts ......................................................................................................

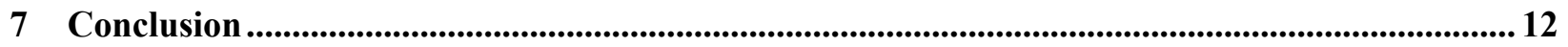

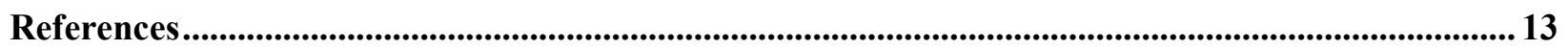

FIGURES

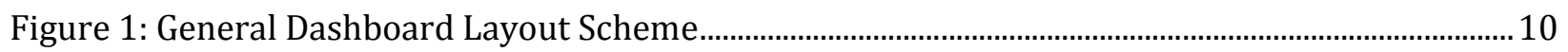

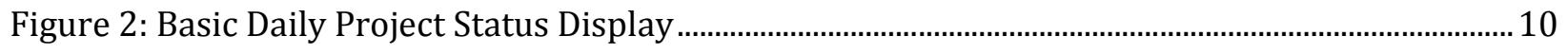

Figure 3: Bullet Chart - Daily Activity Detail .............................................................................................. 11

Figure 4: More Advanced Visualizations ………............................................................................................ 11 


\section{INTRODUCTION}

\subsection{Outage Information and Situation Awareness}

As we have seen from observations during outages so far, to achieve even a single objective often requires access to a collection of information that is not necessarily related, often coming from diverse sources related to various outage, maintenance, and operational functions. This information is often difficult to classify due to large variability, and each recipient must spend some cognitive effort on determining the relevance to his or her job. This information can be one or more key performance indicators (KPIs), it could be quantitative data, or it could be qualitative information that must be "translated" into information relevant to the task at hand.

Most of the activities during an outage require constant monitoring in real-time or near-time, because delays in responding cannot be tolerated without impacting schedules and cost. This is why outage information must be designed to support real-time collective situation awareness, in the same way that operators in the control room need to be constantly aware of the plant's condition to ensure safe and effective operations. Information displayed in the Outage Control Center (OCC) must grab the attention of the OCC staff when it is needed, it must make it easy to spot what is most important in a screen full of data, and it must give all team members the means to understand the status and importance of all conditions and activities and respond without little or no delay. To do this, they require expert visual design to ensure that the displays express measures of performance clearly, accurately, directly, and without distraction.

The need for optimal situation awareness was mentioned in the FY14 AOCC milestone report ( $\mathrm{St}$ Germain et al., 2014) but without mentioning the design, type and quality of information represented in the OCC. Endsley et al. (2003) define situation awareness as follows:

"Situation awareness is being aware of what is happening around you and understanding what that information means to you now and in the future. This awareness is usually defined in terms of what information is important for a particular job or goal. The concept of situation awareness is usually applied to operational situations, where people must have situation awareness for a specified reason, for example in order to drive a car, treat a patient, or separate traffic as an air traffic controller."

Situation awareness involves three levels of awareness that apply in any work domain: 1) the perception of one's environment, 2) comprehension of its meaning, and 3) the ability to project that understanding into the future to anticipate what might happen.

This definition is actually a very accurate description of the requirements for optimal outage performance and it implies that all critical performance measures should be designed to support the execution of individual, as well as collective outage activities. In addition, this means that an information display that is designed to support situation awareness must support all three levels of awareness. Failure in any of these areas will undermine the effectiveness of the information displays and thus reduce overall outage performance. However, the entire weight of responsibility for the success or failure of the information displays does not fall on the display alone. Workers who use the information must thoroughly understand the domain. They have already constructed a mental model of the domain into which the information derived from the display can be rapidly integrated. However, a perfectly designed display will not overcome their lack of expertise and expertise cannot overcome the failures of the display to present information in a way that matches their individual or collective mental model.

Because of the unique cognitive and perceptual requirements posed by the complex information generated during outages, the effective design of an integrated information display requires an understanding of human factors in general, and visual communication in particular - what works, what doesn't, and why. This involves an analysis of the nature, role, and composition of the discrete components of the visual elements in the displays. This is a necessary element in the analysis of situation awareness, due to the very nature of the processes of representation, communication and interpretation of 
information in all work domains. In fact, the semantic content of information artifacts in the OCC is so high that it should be treated as a complex, hierarchical architecture of meanings, expectations, targets, values, and measures.

As indicated above, optimum situation awareness requires knowledge of, for example, current outage performance parameters and the normal value of those parameters, the difference between current values and normal values, the past state of the process, and the predicted future state of the process. Situation awareness is maximized by integration of this information, and is thus critical when the OCC team members are confronted by a complex and changing situation. It is directly related to individual worker and joint team performance, and is especially important during abnormal conditions (e.g, emergent conditions such as equipment damage, leaks, releases, etc.) when personnel are required to identify situations and problems not covered by normal procedures, make correct diagnoses of faults, and decide on a path forward. Research has shown that the way in which information about the dynamic environment is represented in a person's mental model, plays a significant role in anticipation of certain events, and thus also affects a conscious attention and search for information. There is also common agreement that the work situation in complex industrial environments is characterized by high information content, which, if not managed properly, may contribute to excessive mental workload, and hence operator error.

It must be emphasized that more information is not necessarily better for optimal performance, because too much information can cause "cognitive clutter" and may interfere with effective response and appropriate mitigation. Methods of providing raw data to OCC team members are currently simplistic and do not exploit the potential of effective visual communication. Additionally, there is much more data available that is not typically evaluated, because methods have not yet been developed to process and integrate this information into something meaningful. We know that the schemata that make up a person's mental model are constructed through perception, attention, pattern matching, analysis, synthesis, and metacognitive processes. These are all directly associated with the process of semiosis (the process of engaging the senses in the interpretation of signs as referring to their objects, in an attempt to obtain meaning from visual representations), but situation awareness analysis techniques have so far not included this perspective.

To understand how presentation of information in the OCC affects human performance and thus overall outage performance, we need to understand how information perceived by the worker acquires meaning in specific situations. More specifically, we need to ask how individual signs in OCC displays are distinguished from, and also related to, the total context, that is, how does the individual worker and also the team decide where to focus their attention, whether regarding the external world (the plant) or regarding their own interior world (mental model)? We also need to know what contributes to the perceptual salience of the information. How does the sign modify the worker's internal mental organization, that is, the mode of interpretation of the sign? These questions fall squarely in the domain of semiotics (that is, the study of signs and meaning). Measures of optimal situation awareness therefore need to include an analysis of the actual information that the OCC members deal with: location, type, duration (transience), frequency (repetition), structure, format, accuracy, origin, etc. (Hugo, 2005).

\subsection{A semiotic approach to the design of an advanced OCC Information Display}

It is not the purpose of this paper to present an extensive discussion on semiotics, which is a rather arcane subject for most people. However, this is such a useful paradigm to explain the structure of visual communication in the OCC, that a brief introduction will provide the reader an understanding of the underlying premise of the proposed design approach.

Although more formal definitions may be found, semiotics can simply be called the study of signs and meaning. The concept is particularly relevant to work domains characterized by electronic communication media, because they are so rich in different forms of visual representation. The field of semiotics formulated the notion of the semiotic sign that serves as the theoretical basis for any perceptive phenomenon, be it internal or external. The visual nature of any user interface and its diversity and 
complexity of signs immediately implies that it requires more than just a superficial investigation of the role and meaning of signs.

According to one of the models in semiotic theory, a sign is defined as a binary relationship between the signifier and the signified, where the signifier is the form that the sign takes, something that can be perceived by the senses, for example, an icon in the operator interface that represents a pump. The signified is the concept to which it refers, which can be a mental construct (for example, "start the sequence to drain the tank"), or something concrete in the world, such as a physical pump, to which the icon refers. (Chandler, 1994, p. 36-42).

The interaction between the signifier and the signified is a perceptive-cognitive process of converting the visual representations ("signs") into meaning. This sense-making process is known in semiotics as "semiosis" and this is an everyday challenge for all humans who unconsciously and continually are confronted by the question: "how do I know that what I see means what I think it does?"

The theory further explains that the relation of the signifier to its signified object can be either iconic (some kind of visual similarity), indexical (some kind of causal relation), or symbolic (some kind of convention or rule). In advanced control rooms using digital displays, the icon is a common visual artifact where the appearance of the icon usually stands in a one-to-one relationship with the thing that it represents, for example, an icon of a pump on a mimic diagram is a rough visual representation of what a pump actually looks like and it represents the a flow or transfer function. Mimics often also employ indexical indicators of various kinds to represent causality, for example, colors or shapes to indicate system status, or lines and arrows to represent flow.

The Advanced OCC (AOCC), like the main control room, is a combination of physical and virtual (electronic) constructs. It is governed and defined by its own rules and laws of existence, and it can thus be seen as an expression of a set of known semiotic systems. In semiotic terms, the AOCC is simultaneously an index and an icon (i.e., representational as well as causal). When we delve deeper into its structure, the AOCC may be defined as a high order, complex sign system consisting of a web of many other complex signs. In this semiotic space, all signs are coordinated to work together to express a particular model of the world. Quite literally, nothing is without meaning or without an intended message in this domain. In this way, the semiotic space of the AOCC is constructed from the synthesis of all possible semantic and syntactic elements.

Ultimately, a semiotic approach to the design of AOCC information supports the cognitive-semantic aspects of sign composition and is thus well suited to the analysis and design of displays in this complex task domain. A coherent taxonomy or framework of structured representations would provide a practical way to ensure consistency and coherence in the display architecture. It should thus be possible to ascertain with a greater degree of accuracy and confidence why, how and when certain display configurations promote and others inhibit situation awareness. An analysis of the semiotic and semantic architecture of the OCC would also help to assess the "semiotic adequacy" of displays, in other words, why a particular instantiation of a display is better to promote situation awareness than another.

\section{Data complexity and the use of information dashboards}

Experience in many industries provides convincing evidence that so-called information dashboards are one of the most effective ways to improve situation awareness. When properly designed, a dashboard can reduce the complexity of massive amounts of disparate pieces of information from various sources and integrate all information on a single display panel.

The real power of an information dashboard lies in its ability to exploit the process of semiosis, that is, the innate human ability to connect how we think with what we see - hence the expression "I see" as a substitute for "I understand." When we make sense of something, we refer to what we have learned as "insight." 
Most of the information generated during an outage is ultimately quantitative in nature and much of this can be expressed as specific measures of performance. The visual display of this quantitative information on a dashboard not only exploits the fact that vision is the primary sensory source of information, but it is also faster, based on bandwidth of input, and richer. This is especially true if information can be presented graphically, because a graph is designed to integrate a number of variables into a single image that communicates one coherent concept. Dashboards may include words (which are often the best or only way to communicate many concepts), but the more we can rely on images to convey the message, the faster that message can be perceived and interpreted. We perceive images in a parallel manner (lots of information all at once), which is much faster than the serial perception of language thus, "a picture is worth a thousand words".

There is little argument about the complexity of AOCC data; therefore, it is necessary to be able to analyze and interpret all of this data to improve the performance of the AOCC. Big data is not just the sheer quantity of data, but instead, it is the complexities and the hidden relationships between data points that characterize a dataset as big data. Analysts employ a process to analyze complex data that conventional statistical methods and business intelligence applications cannot handle. Big data analytics is the process that seeks to uncover hidden patterns in complex data that can then be used to make better and quicker decisions. Within the hidden patterns, we can analyze past performance to improve efficiency and performance in an AOCC.

The primary indicator that will be created during this effort will be a bulk work completion confidence factor. This factor will account for several process parameters that may affect work execution and account for past performance. This factor will be similar to the FICO credit score that many are familiar with. The data that will eventually be included in this factor include:

- Schedule Data (most important and first to be applied)

- $\quad$ \# of activities scheduled vs completed

- Man-hours scheduled vs completed

- \# of activities remaining vs historical completion

- Man-hours remaining vs available resources

- Etc.

- $\quad \#$ of clearance orders outstanding

- $\quad \#$ of persons signed on to clearance orders

- $\quad$ \# of RCA entries per day

- Total daily dose accumulated

- $\quad$ of condition reports submitted

- \# of work orders added to outage scope

- \# of clearance order hung or cleared per day

- Any others that utilities have data available for that may be meaningful

The only coping mechanism available to team members in perceiving and interpreting information is their own cognitive abilities. Although it is true that graphical presentation of information is often better than words or numbers, simply presenting raw data as graphs, as is usually the case at present, does very little to reduce the cognitive burden. Making sense of AOCC data relies almost entirely upon knowledge and experience. Unfortunately for much of the nuclear industry, there will soon be a large loss of knowledge and experience as senior staff retire and are replaced by younger, less experienced staff. Much of this burden could be reduced by applying big data analytical "pre-processing" techniques before 
displaying the data. This calls for a scientific method called Visual Analytics. This method combines information visualization techniques with data analysis and computational transformation techniques, which support analytic reasoning, and thus the sense-making process (Keim et al., 2010). Visual analytics creates a powerful, direct interface between user and machine, amplifying human cognitive capabilities in five basic ways:

- It increases human cognitive resources by providing an external visual resource to expand human working memory;

- It simplifies and reduces the need to search for information by representing a large amount of integrated and synthesized data in a small space;

- It enhances the observer's ability to recognize patterns, for example by organizing information spatially, by time relationships, by function, etc. This also supports easy perceptual inference of relationships that are otherwise more difficult to deduce;

- It supports situation awareness by simplifying the monitoring of a large number of potential events;

- Finally, visual analytics helps to create a coherent, consistent, interactive, manipulable medium that, unlike static, raw data diagrams, enables the exploration of the AOCC information space.

Ultimately, visual representations of information help us work around a fundamental limitation that is built into our brains - a limited capacity to store information for immediate recall. Although we have tremendous long term memory capacity, working memory (also known as short-term memory) is extremely limited. Graphical representations of quantitative information allow people to store the entire pattern formed by the shape of the graph (histogram, trend graph, pie chart, etc.) as a single chunk of memory. This means that we can store much more information within the limitations of short termmemory than we could if the values were written as numbers. Simply by encoding information visually, our ability to think about it can be significantly expanded by making more of it available at any one moment.

Reiterating the benefits of visual analytics mentioned above, we can see that by placing all of the information needed to support decision-making during outages on a single display, it becomes possible to (1) reduce the need to rely on short-term memory, (2) reduce the need to supplement short-term memory by printing paper copies, (3) reduce the need to obtain information from other sources, (4) let the dashboard serve as an external form of memory, and (5) reduce the need to move the information into long term memory and instead enable moving it in and out of short term memory at lightning speed as you make sense of it.

\section{Purpose and Advantages of an Outage Dashboard}

The purpose of an outage dashboard is the visual display of the most important information needed to achieve one or more objectives of the outage. This information will fit entirely on a single display panel so it can be monitored at a glance by all OCC team members. Some or all of the information could also be made available for dissemination to remote locations and handheld devices.

The outage information on a dashboard is presented as a combination of text and graphics, but with an emphasis on graphics. As explained above, dashboards are highly graphical, not to create "pretty pictures", but because graphical presentation, handled expertly, can often communicate with greater efficiency and richer meaning than text alone. The design process must determine optimal ways to present the information so that it can be perceived quickly and enable OCC team members to easily extract the correct and most important meanings from it.

In addition to the advantages of efficient communication, a well-designed dashboard should also help the whole team to manage the bow wave of work. While the obvious approach is to avoid bow wave work and not to continually have to recover from its effects, information should be synthesized in a way that 
will enable operations to be ready with clearances and resources to support testing activities, and to release work based on priority and schedule (e.g., long duration work released first). This cannot be a one-sided approach and all disciplines should be consulted on how to improve production and barriers that can be removed - work has to be "READY-READY". This includes factoring emergent work into initial schedule loading, down to the crew level based on experience from previous outages.

The most noteworthy advantages of a well-designed dashboard, that is, one that applies visual analytics techniques, are:

- Reduce information complexity

- Reduce the number of alert conditions and instead improve the differentiation of alerts

- Aggregate and integrate disparate information

- Include analysis of past performance

- Ensure visual salience (the ability to focus on the right things for the right reason at the right time)

- Ensure a match between information and its visual representation (the right kind of graphic for the right kind of data)

- Direct expression of quantitative measures of performance

- Ensure coherence and understanding of overall context of activities

\section{Dashboard Functional and Physical Characteristics}

Not all of the information described above will be equally important and it must be ranked and rationalized before embarking on the design of the dashboard. The following principles, some of which are also found in St. Germain, 2015, will guide the ranking:

- Which items are needed for immediate decision-making?

- Is there a need to see discrete data all the time?

- Is the discrete information alone valuable to the performer to accurately make the correct decision?

- Can the discrete data be displayed using color coding to provide additional information about the state or status of the process or system?

- Which items can be omitted because they do not support decision-making?

- What critical decisions are required by the performer?

- Which items will encourage active thinking about the data, not just passive reaction to alerts?

- Which items will provide team members a simple means to respond?

- Which collection of items will provide a common picture for the whole team?

- Which items will support projections for proactive responses (situation awareness)?

- Do the selected items jointly and individually match the team members' mental model?

- Can the data be displayed using a trending graphical display? Is this more a useful form?

- Will a digital or analog display or combination of the two improve understanding by the performer?

In addition to the functional requirements, consideration must also be given to the physical configuration of the displays, within the context of the physical OCC and also physical environments and workspaces outside the OCC. 
The ideal dashboard can be characterized in terms of the hardware requirements as well as the visual display properties of the user interface. The following items, which include some notable requirements described by St. Germain (2015), will influence the overall design of the dashboard:

- The size and number of monitors must be determined, based upon an analysis of tasks, workflow and physical work spaces.

- The design of the user interface must follow well-established human factors and visual communication guidelines (e.g. Few, 2013).

- The amount of information that it displays must not be overwhelming.

- Each item of information must be displayed in a way that can be quickly examined to assess outage performance, both in terms of an appropriate graphical medium and direct measure of performance (for example, percentage off target).

- Measures must be expressed with enough context (for example, comparisons to targets, averages, and previous periods) for their meanings to be clear.

- Color must be used sparingly and only highlights used for salient items, so that it is easy to spot those items that require attention by, for example, scanning for red.

- The most important items (e.g., the KPI set) must be placed in the prominent position of the screen the upper left.

- Although the dashboard alerts the person using it to problems, it must not automate a response, but require human intelligence to be engaged in the process and initiate responses.

- Responses must be made easily, such as by clicking on the name of an OCC member who is experiencing difficulty to send an instant message. Because the information can be updated frequently (hours, minutes, or even seconds), when a close examination is desired, updates could be temporarily halted to remove the distraction of changing data.

- Only what is necessary must appear on the dashboard. It must not be cluttered or distractingly decorated. It should not need any instructions for how to use it.

Any additional requirements must be determined through a functional requirements analysis, task analysis, and careful consideration of usability requirements, in terms of guidance provided by ISO 924111 (ISO, 1998).

These criteria can be reduced to a simple framework that helps to identify the required items.

For each item the following questions must be answered positively:

1. Can the information be displayed in a way that makes it actionable? (e.g., does the metric go up, down, flat or off-target and is the required action obvious?)

2. Does the item ensure common interpretation? (i.e., will all team members recognize what this metric means?)

3. Is the metric source transparent? (i.e., how was the metric derived?)

4. Are the data accessible and credible? (i.e., can data be acquired with modest effort from a source that people trust?) 
Since all information items indicated above are quantitative in nature, it will be possible to use a combination of the following graphical representations:

1. Bullet graphs (vertical or horizontal) - for parameters that can be tracked for planned and actual performance against measures such as "below expectation, acceptable, exceed expectation".

2. Line graphs - for measures that have a temporal component, i.e., tracked for performance over time.

3. Histograms - for parameters with a volume or quantity component.

4. Bar graphs with markers for targets and limits - for parameters showing variable quantities (speed, volume, count, etc.)

5. Heat maps - for parameters that deal with abstract or physical areas of variable concentration (e.g. radiation zones, emergent issues, etc.)

6. Colored flags (icons, symbols or indexes) - for status parameters (e.g. red/yellow/green).

7. Numerical fields - Only where discrete values are important. These may be in the form of a table.

8. Alerts - to indicate parameters that exceed a threshold (similar to type 6 above)

\section{Selection of key performance indicators}

Although there are minor differences among utilities and plants regarding the primary measures of planned outage performance, the following appear to be accepted best-practice indicators (IAEA, 2006a):

- Total outage performance, measured in terms of duration, deviation from critical path, number of planned activities, hours/days required for activities, deviation from daily schedule, etc.

- Outage financial budget

- Outage dose budget

- Contract work execution (modifications, tests, refurbishment, replacements, etc.)

- Maintenance work status (mechanical, electrical, I\&C)

- Spare parts and materials availability

- Refueling status

- Human performance (fitness for work, injuries, error, procedures)

- Plant safety - individual radiation dose, personnel contamination events, etc.

- Protected equipment isolation status and unplanned engineered safety system actuations

- Workload utilization

- Hazards (fire, releases, spills, leaks, dropped items)

- Nuclear safety events

- Operation limits and conditions entries

- Emergent issues (severity and impact of unplanned work, rework, damage, foreign material intrusions, etc.)

- Unplanned risk challenges 


\section{Basic Dashboard Design Concepts}

The visual structure can serve as a navigational mechanism for the user. It shows where to start, and where to go next. Dashboard content should be organized in a way that reflects the nature of the information and that supports efficient and meaningful monitoring. Information cannot be placed just anywhere on the dashboard, nor can sections of the display be sized simply to fit the available space. Items that relate to one another should usually be positioned close to one another. Important items should often appear larger, thus more visually prominent, than less important items. Items that ought to be scanned in a particular order ought to be arranged in a manner that supports that sequence of visual attention.

Key considerations for the dashboard will include the following:

- Timeliness: How frequently will the data in the dashboard be updated?

- Aesthetic value: How important is it that the dashboard looks attractive, or can it be purely utilitarian?

- Mobility: Do users need to access the information on the go? In which case, can the dashboard and/or some or all of its contents be disseminated to portable devices?

- Connectivity: Does the dashboard need to connect to live data sources?

- Data detail: Will the dashboard offer an ability to drill down to see more context?

- Data density: How information-rich do views of the data need to be?

- Interactivity: How will users benefit from interacting with the dashboard?

- Collaboration: Is it important for OCC members to be able to easily share and collaborate on the dashboard?

- Is it necessary for software applications to be embedded in the dashboard display (e.g. Excel, P6, etc.)?

- Does the dashboard need filters to reduce, expand or collapse detail in specific groups?

Ultimately, the dashboard should be designed to convey a lot of information without being overwhelming. This will require rigorous attention to five elements:

1. Information design - selection, classification and validation of information elements

2. Graphic design - selection, semiotic evaluation and design of graph types to match information types

3. Interface design - layout, typography, color, and semiotics

4. Interaction design - selection and design of navigation schemes and interactive features

5. Usability testing - ensuring that specific users are able to use the dashboard with effectiveness, efficiency, and satisfaction in defined contexts.

These principles are not meant to enforce any rigid design and layout constraints. Rather, in keeping with a logical form-fit-function approach, an analysis of the data as well as functions and tasks should precede any attempt to design a dashboard. Nevertheless, it is recognized that a significant amount of 
research and experience is available to inform the design and we intend to take advantage of proven applications, and also learn from mistakes made in the past (Few, 2004).

The following mockup examples illustrate the basic concepts that could be pursued in the design.

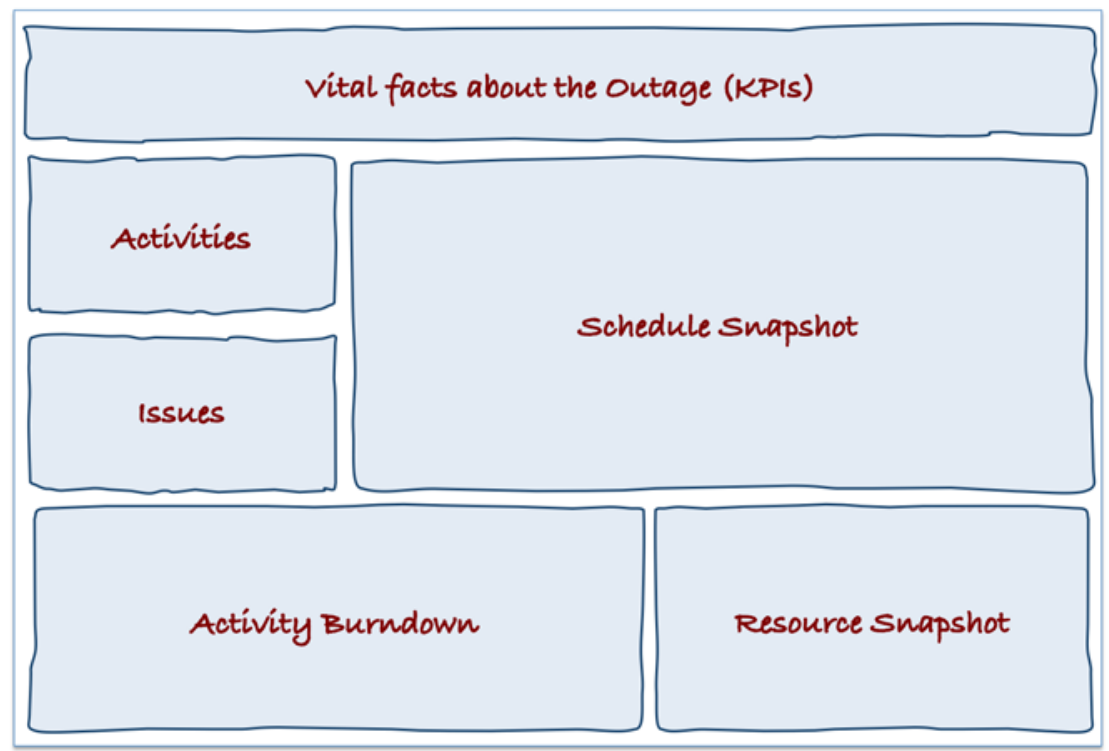

Figure 1: General Dashboard Layout Scheme

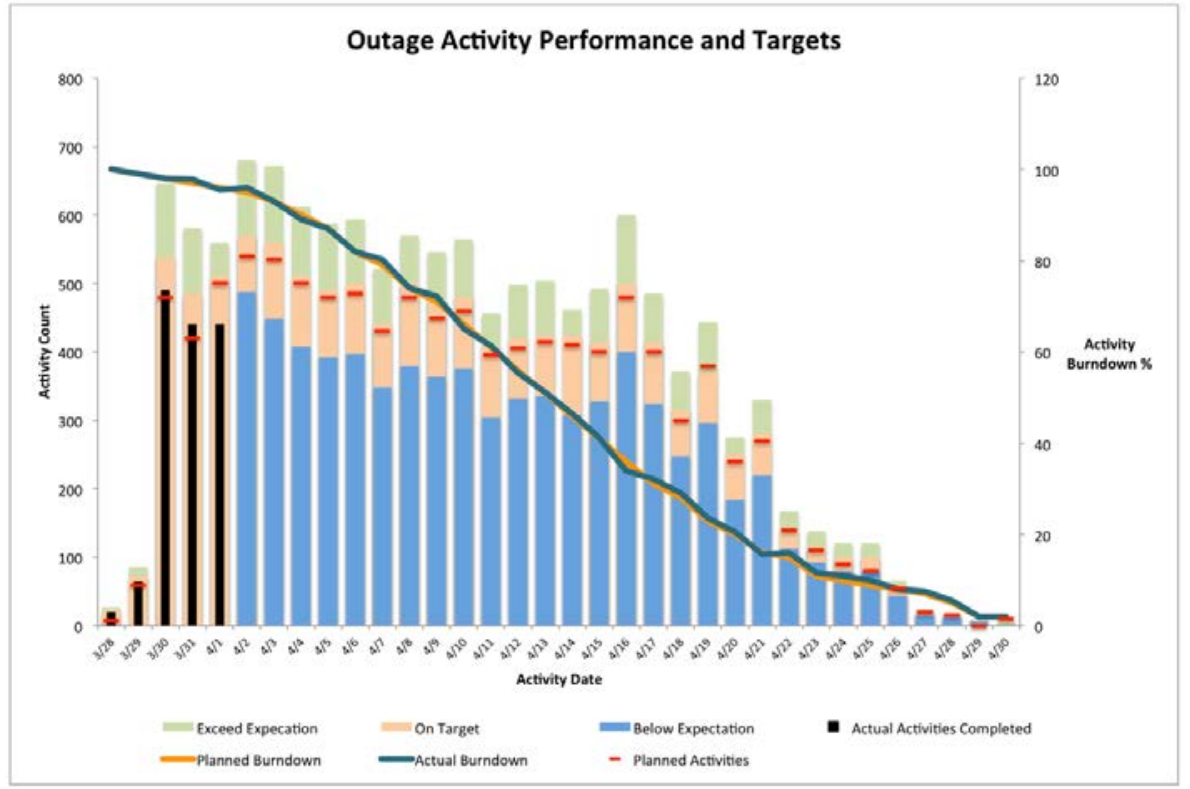

Figure 2: Basic Daily Project Status Display 


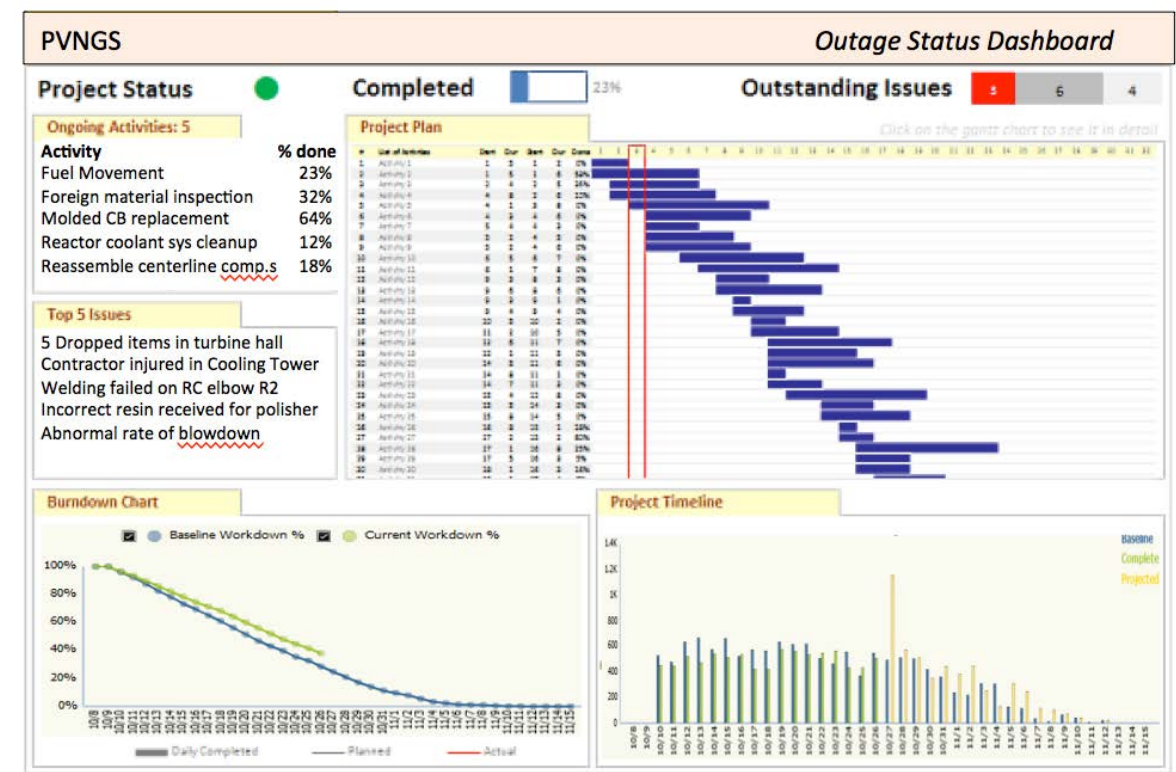

Figure 3: Bullet Chart - Daily Activity Detail

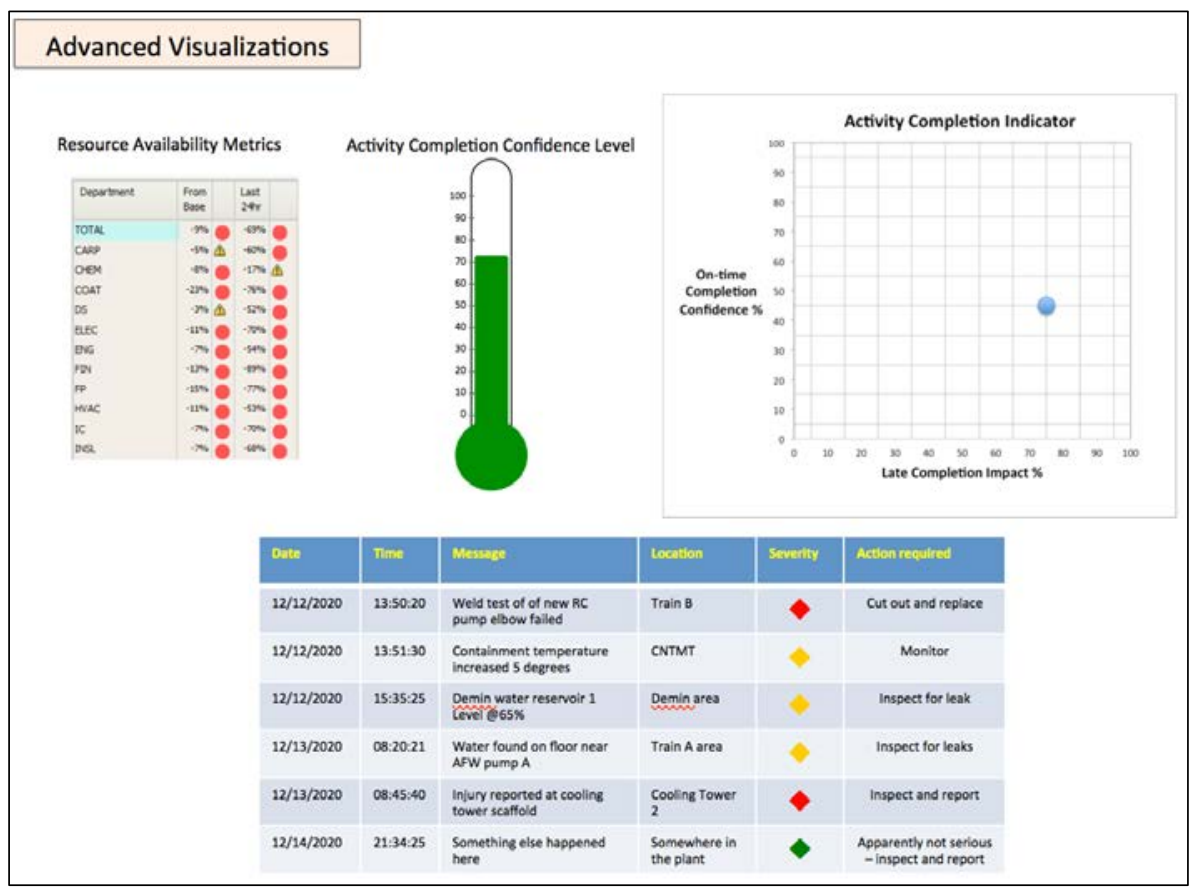

Figure 4: More Advanced Visualizations 


\section{Conclusion}

While the means and criteria for measurement of outage performance have been reasonably well established, it is much more difficult to measure human performance in the AOCC and even more difficult to relate that performance to overall outage performance. All existing methods of determining the nature of human behavior and the parameters of task performance attempt to explain the reasons for deficient performance and human error. None of these methods explains the process whereby a person observes and interprets the signs in the user interface and decides to perform a specific action. They fail to acknowledge that what really happens between a human and an information display is not simply "interaction". It should rather be seen as a process of communication where the accuracy of information, and thus the correctness of the person's performance, is directly influenced by decisions made by designers of the information dashboard.

An important observation made by Keim at al (2010) is that all grand challenge problems of the 21 st century, such as climate change, energy, finance, health or security crises, require the exploration and analysis of very large and complex data sets which can neither be done by the computer nor the human alone. This is certainly true of AOCC data, as well and by understanding the semiotic nature of the dashboard coupled with the underlying data complexity, designers should be able to make less arbitrary decisions about the overall hierarchical information architecture. This would include decisions about choosing among different graph types, and the allocation of specific tasks and decisions to specific graphic representations.

It is important to understand that OCC team member performance cannot be divorced from the environment, which includes the information artifacts. Performance is triggered by perceptual or cognitive stimuli in the environment, and tasks are executed within the environment by interacting with various artifacts, which in turn produces other stimuli, giving rise to an endless process. Intimately interwoven with this is the process of semiosis, which has an inescapable effect on performance. In other words, by understanding the relationship between a sign, its meaning and the overall outage context, we will have a clearer understanding of the reasons why different representational modalities are better than others to convey operational information in specific contexts, thereby improving the chances of achieving the required performance (Bertin, 1983 p.9).

This paper has barely scratched the surface of the synergy between the scientific fields of semiotics, visual analytics, situation awareness, and human-system interaction, but past experience in many OCCs provide compelling evidence that existing ways of providing outage performance information is suboptimal. The approach described in this paper will make it possible to determine with greater accuracy what contributes to the perceptual salience of the information, how specific design elements may contribute to effective communication, and how a well-designed AOCC dashboard that incorporates data analysis may contribute to overall outage performance. 


\section{References}

[1] Bertin, J. (1983). Semiology of Graphics. Redlands, CA: Esri Press.

[2] Chandler, D.L (1994). Semiotics for Beginners. London: Routledge.

[3] Daniel Keim, Jörn Kohlhammer, Geoffrey Ellis and Florian Mansmann (Eds) (2010).

Mastering the Information Age - Solving Problems with Visual Analytics. Downloaded from Eurographics Digital Library, http://diglib.eg.org

[4] Endsley, M., Bolte, D. \& Jones, D. (2003) Designing for Situation Awareness: An Approach to User-Centered Design. CRC Press: Boca Raton..

[5] Few, S. (2004). Show Me the Numbers: Designing Tables and Graphs to Enlighten. Oakland, Calif: Analytics Press.

[6] Few, S. (2013). Information Dashboard Design - Displaying data for at-a-glance monitoring. Oakland, Calif: Analytics Press.

[7] Hugo, J. (2005). The Semiotics of Control Room Situation Awareness. In Proceedings of CybErg 2005 - The Fourth International Cyberspace Conference on Ergonomics, Johannesburg, South Africa (2005).

[8] IAEA-TECDOC-1490 (2006a). Indicators for Management of Planned Outages in Nuclear Power Plants. International Atomic Energy Agency:Vienna, Austria.

[9] IAEA TECH REPORT 449 (2006b). Management Strategies for Nuclear Power Plant Outages. International Atomic Energy Agency:Vienna, Austria.

[10] ISO 9241-11 (1998). Ergonomic requirements for office work with visual display terminals (VDTs) -- Part 11: Guidance on usability. International Organization for Standardization.

[11] St. Germain, S., Farris, R., Whaley, A., Medema, H., \& Gertman, D. (2014). Guidelines for implementation of an Advanced Outage Control Center to improve Outage Coordination, Problem Resolution, and Outage Risk Management. (INL/EXT-14-33182). Idaho National Laboratory, Idaho Falls, ID.

[12] St. Germain, S., Farris, R., \& Thomas, K. (2015). Development of Improved Graphical Displays for and Advanced Outage Control Center, Employing Human Factors Principles for Outage Schedule Management. (INL/EXT-15-36489). Idaho National Laboratory, Idaho Falls, ID. 